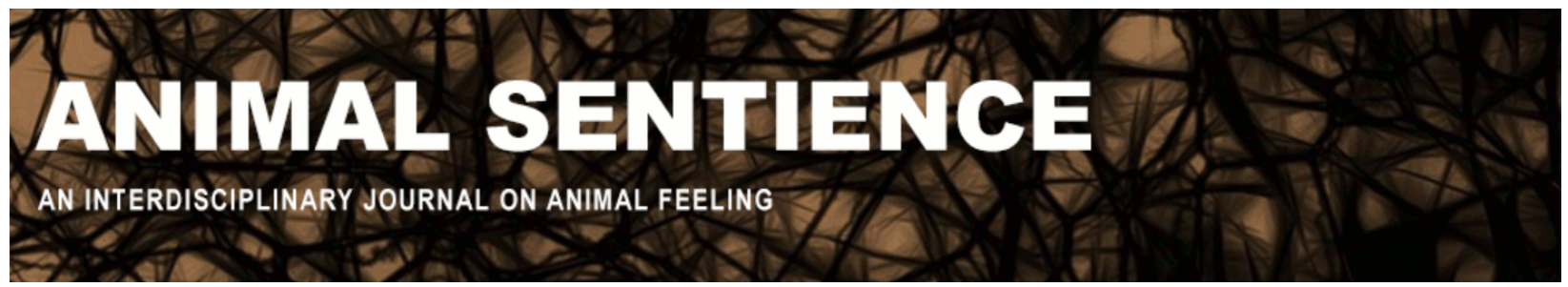

Bar-Hen-Schweiger, Moran and Henik, Avishai (2019) Intelligence as mental manipulation in humans and nonhuman animals. Animal Sentience 23(31) DOI: 10.51291/2377-7478.1409

Date of submission: 2019-02-09 Date of acceptance: 2019-02-18 (c) 


\title{
Intelligence as mental manipulation in humans and nonhuman animals
}

Commentary on Chapman \& Huffman on Human Difference

\author{
Moran Bar-Hen-Schweiger and Avishai Henik \\ Department of Psychology, and Zlotowski Center for Neuroscience \\ Ben-Gurion University of the Negev, Beer Sheva, Israel
}

\begin{abstract}
Chapman \& Huffman review and evaluate various aspects of the notion of human superiority. In this commentary we focus on intelligence and suggest a biologically based view of intelligence applicable to humans and non-human species alike. "Mental manipulation" (e.g., mental transformations, rotations, perspective-taking), an extension of object manipulation, provides a continuous, biologically based concept for studying intelligent behavior in humans and other species and challenges the notion of human superiority.
\end{abstract}

Moran Bar-Hen-Schweiger investigates mental manipulation in intelligence assessment. She is also interested in studying the implications of mental manipulations in the field of neuropsychology. Website

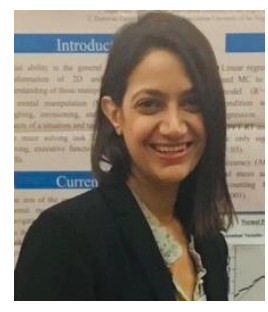

Avishai Henik, Distinguished Professor of cognitive neuropsychology at the Department of Psychology, Ben-Gurion University of the Negev, Israel, is author of over 250 publications in the general area of cognitive neuroscience: attention, cognitive control, numerical cognition, and synesthesia. Website

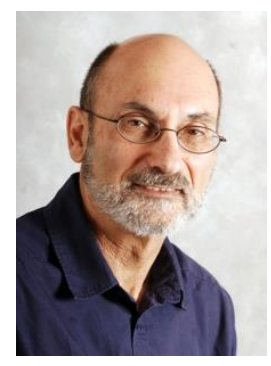

"The difference in mind between man and the higher animals, great as it is, certainly is one of degree and not of kind" (Darwin, 1871, p. 105).

Whereas Darwin's (1871) doctrine of continuity among human and non-human species is well established and supported by evidence, the claims regarding human superiority have not vanished. Chapman and Huffman (2018) review and evaluate the common statements about human superiority and offer a historical, sociological and cognitive perspective on these deeprooted beliefs. We agree that the human-animal distinction in intellectual capabilities is inadequate. Intelligent behavior in humans and animals is typically defined among non-human species in an overly anthropocentric manner, emphasizing skills characteristic of Homo sapiens. For example, intelligence in humans has been based on the human ' $\mathrm{g}$ ' factor, rather than a ' $\mathrm{G}$,' representing observed skills among other species. Similar distinctions have limited the generalizability of the concept of intelligence and its application in biological and cultural contexts (see also Bar-Hen-Schweiger et al., 2017; Burkart et al., 2016; Sternberg, 2017). 
The continuous nature of intelligence has been described in the past by Piaget (1952, 1971). Sternberg (2017, p. 45) wrote "an appropriate way to look at the intelligence of any organism is to look at how well it adapts to the range of environments it confronts." Viewing intelligence as a capacity necessary for survival in humans and animals alike offers an opportunity for cross-disciplinary, cross-species study free of preconceived, hierarchical categories. We propose a description of intelligence in which cognitive functions are an extension of biological skills across species that evolved from object manipulation into mental manipulation (Bar-HenSchweiger and Henik, 2019, submitted) in response to various environmental conditions. Species facing a variety of environmental challenges evolve bigger brains and increase the potential for developing a larger repertoire of domain-specific skills during maturation (Reader et al., 2011). Object manipulation capabilities increase adaptation and support adjustment to environmental demands (Hayashi et al., 2006).

We have proposed that mental manipulation is an overarching principle reflecting the ability to perform transformations, translations, re-combinations, projection, and prediction, in infinite ways. Mental manipulation involves progressive abstraction, so that the rotated object no longer needs to be present; it, and actions on it, can be imagined. An early study of quantifying abstract mental manipulation is the famous one by Shepard and Metzler (1971), who timed mental movement of imagined object rotations. Another example is perspective-taking, the ability to adopt a viewpoint of a scene different from one's own. Mental manipulation enables an organism to consider various consequences of these operations and then select the best strategy for action. It is the hallmark of the human species, but it is not unique to Homo sapiens. It constitutes a value-free, unifying dimension along which humans no longer occupy a unique and exclusive position. The covariation of object manipulation skills with brain size and other cognitive functions (Heldstab et al., 2016) may provide fertile ground for cross-disciplinary research efforts in several fields, such as neuroscience, neuropsychology, biology, and sociology, leading to anatomically informed measurements of skills.

New instruments will be needed to measure this unifying dimension and compare different species as inhuman, and honeyguide birds' collaboration in locating honey using vocal communication (Spottiswoode et al., 2016). Such collaboration requires considerable planning and appreciation for the mutual goals of each partner. Can humans, in this context, be considered more intelligent?

Even behaviors thought to be uniquely human, such as compassion and deception, require mental manipulation to appreciate the mental state of another being and have been observed also in animals (Byrne and Corp, 2004; Marino, 2017). Mental manipulation provides an ecologically valid, unifying dimension of cross-species intelligent behavior. 


\section{References}

Bar-Hen-Schweiger, M., \& Henik, A. (2019). The transition of object to mental manipulation: Beyond species-specific view of intelligence. Manuscript submitted for publication.

Bar-Hen-Schweiger, M., Schweiger, A., \& Henik, A. (2017). G but not g: In search of the evolutionary continuity of intelligence. Behavioral and Brain Sciences, 40, e199.

Burkart, J. M., Schubiger, M. N., \& van Schaik, C. P. (2016). The evolution of general intelligence. Behavioral and Brain Sciences, 40, e195.

Byrne, R. W., \& Corp, N. (2004). Neocortex size predicts deception rate in primates. Proceedings of the Royal Society B: Biological Sciences, 271(1549), 1693-1699.

Chapman, C. A., \& Huffman, M. A. (2018). Why do we want to think humans are different? Animal Sentience 23(1).

Darwin, C. (1871). The descent of man, and selection in relation to sex (2 vols.). London, England: John Murray.

Hayashi, M., Takeshita, H., \& Matsuzawa, T. (2006). Cognitive development in apes and humans assessed by object manipulation. In T. Matsuzawa, M. Tomonaga, \& M. Tanaka (Eds.), Cognitive development in chimpanzees (pp. 395-410). Tokyo, Japan: Springer.

Heldstab, S. A., Kosonen, Z. K., Koski, S. E., Burkart, J. M., Van Schaik, C. P., \& Isler, K. (2016). Manipulation complexity in primates coevolved with brain size and terrestriality. Scientific Reports, 6, 24528.

Marino, L. (2017). Thinking chickens: a review of cognition, emotion, and behavior in the domestic chicken. Animal Cognition, 20(2), 127-147.

Piaget, J. (1952). The origins of intelligence in children. New York: International Universities Press.

Piaget, J. (1971). Biology and knowledge. Chicago: University of Chicago Press.

Reader, S. M., Hager, Y., \& Laland, K. N. (2011). The evolution of primate general and cultural intelligence. Philosophical Transactions of the Royal Society B: Biological Sciences, 366(1567), 1017-1027.

Shepard, R. N., \& Metzler, J. (1971). Mental rotation of three- dimensional objects. Science, 171, 701-703.

Spottiswoode, C. N., Begg, K. S., \& Begg, C. M. (2016). Reciprocal signaling in honeyguide-human mutualism. Science, 353(6297), 387-389.

Sternberg, R. J. (2017). It's time to move beyond the "Great Chain of Being". Behavioral and Brain Sciences, 40, e219. 